\title{
Relativistic filamentary equilibria
}

\author{
M. GE D A L I N ${ }^{1}$, A. S P I T K O V S K Y ${ }^{2}$, M. ME D V E D E V ${ }^{3}$, \\ M. B A L I K H I N ${ }^{4}$, V. K R A S N O S E L S K I K H H , A. V A I V A D S \\ and S. PER R I ${ }^{7}$ \\ ${ }^{1}$ Ben-Gurion University, Beer-Sheva, Israel \\ (gedalin@bgu.ac.il) \\ ${ }^{2}$ Princeton University, Princeton, USA \\ ${ }^{3}$ Department of Physics and Astronomy, University of Kansas, Lawrence, KS 66045, \\ USA and Niels Bohr International Academy, Niels Bohr Institute, \\ University of Copenhagen, 2100 Copenhagen K, Denmark $\dagger$ \\ ${ }^{4}$ ACSE, University of Sheffield, Sheffield, UK \\ ${ }^{5}$ LPCE/CNRS, Orleans, France \\ ${ }^{6}$ Swedish Institute of Space Physics, Uppsala, Sweden \\ ${ }^{7}$ ISSI, Bern, Switzerland
}

(Received 28 November 2009 and accepted 23 January 2010,

first published online 18 February 2010)

\begin{abstract}
Plasma filamentation is often encountered in collisionless shocks and inertial confinement fusion. We develop a general analytical description of the two-dimensional relativistic filamentary equilibrium and derive the conditions for existence of potential-free equilibria. A pseudopotential equation for the vectorpotential is constructed for cold and relativistic Maxwellian distributions. The role of counter-streaming is explained. We present single current sheet and periodic current sheet solutions, and analyze the equilibria with electric potential. These solutions can be used to study linear and nonlinear evolution of the relativistic filamentation instability.
\end{abstract}

\section{Introduction}

Filamentary instability studies have greatly intensified during the last decade, due to the role they are believed to play in the generation of magnetic fields (Pegoraro et al. 1996; Medvedev and Loeb 1999) and formation of shocks in essentially unmagnetized interstellar plasma penetrated by a relativistic jet ejected from a powerful source, like a gamma-ray burst (Lyubarsky and Eichler 2006; Medvedev 2007; Spitkovsky 2008; Nishikawa et al. 2009). Linear stage of the filamentary development has been extensively studied for various distributions of counter-streaming beams in a wide range of parameters, analytically and numerically (Pegoraro et al. 1996; Yoon 2007; Schaefer-Rolffs and Tautz 2008; Bret 2009). Numerous full-particle simulations have been performed to study the filament growth and merging (Silva et al. 2003; Frederiksen et al. 2004; Hededal and Nishikawa 2005; Spitkovsky 2008; Nishikawa et al. 2009). Yet, there were almost no attempts to analytically construct a stationary filamentary equilibrium, in a way, similar to

$\dagger$ Also at the RRC 'Kurchatov Institute', Moscow 123182, Russia 
the building of a Harris current sheet. Recently, an attempt to derive a GradShafranov-type equation for such equilibrium has been made (Mart'yanov et al. 2008). In doing this, the authors failed to make one of their basic assumptions consistent with Maxwell equations, thus losing an important constraint, which has to be satisfied. In the present paper we develop a general analytical description for two-dimensional filamentary equilibria using the technique outlined in Balikhin and Gedalin (2008). We show that counter-streaming is necessary for the existence of spatially periodic filamentary structure. Fine-tuning, necessary to maintain such equilibria in the presence of four species, is presented.

\section{2D stationary filamentary structure}

Let $x$ and $y$ be the coordinates on the 2D domain, with $z$ being the out-of-plane direction. We consider a $2 \mathrm{D}$ structure of the kind $B_{z}(y)$ and $E_{y}(y)$, where $B_{z}$ and $E_{y}$ are the components of magnetic and electric fields. In principle, there may be an arbitrary number of charged particle species streaming along $x$ and producing the fields. For all these particles, $v_{z}=0$. In what follows, $c \equiv 1$. In this representation the filaments are, in fact, current sheets.

If everything depends on $y$ only, then there are three integrals of motion

$$
\begin{aligned}
& P=p_{x}+q A=m \gamma v_{x}+q A, \\
& H=\sqrt{\boldsymbol{p}^{2}+m^{2}}+q \phi=m \gamma+q \phi,
\end{aligned}
$$

and $p_{z}$, where

$$
E_{y}=-\frac{\partial \phi}{\partial y}, \quad B_{z}=-\frac{\partial A}{\partial y} .
$$

If we express everything in terms of $\boldsymbol{u}=\boldsymbol{p} / m$ and $\gamma^{2}=1+\boldsymbol{u}^{2}$, the integrals of motion can be written as

$$
U=u_{x}+(q / m) A, \quad h=\gamma+(q / m) \phi, \quad u_{z} .
$$

For each species $s$, the distribution function, should be a function of these three integrals of motion:

$$
\begin{aligned}
f_{s}\left(y, u_{x}, u_{y}, u_{z}\right) & =f_{s}\left(U_{s}, h_{s}, u_{z}\right), \\
U_{s} & =u_{x}+\left(q_{s} / m_{s}\right) A, \\
h_{s} & =\gamma_{s}+\left(q_{s} / m_{s}\right) \phi .
\end{aligned}
$$

The current and charge density are

$$
\begin{aligned}
& j_{x}=\sum_{s} q_{s} \int v_{x} f_{s}\left(U_{s}, h_{s}, u_{z}\right) d^{3} \boldsymbol{u}=\sum_{s} q_{s} \int \frac{u_{x}}{\gamma} f_{s}\left(U_{s}, h_{s}, u_{z}\right) d^{3} \boldsymbol{u}, \\
& j_{y}=\sum_{s} q_{s} \int v_{y} f_{s}\left(U_{s}, h_{s}, u_{z}\right) d^{3} \boldsymbol{u}=\sum_{s} q_{s} \int \frac{u_{y}}{\gamma} f_{s}\left(U_{s}, h_{s}, u_{z}\right) d^{3} \boldsymbol{u}=0, \\
& \rho=\sum_{s} q_{s} \int f_{s}\left(U_{s}, h_{s}, u_{z}\right) d^{3} \boldsymbol{u}
\end{aligned}
$$


and Maxwell equations read

$$
\begin{aligned}
& \frac{d^{2} A}{d y^{2}}=-4 \pi j_{x}, \\
& \frac{d^{2} \phi}{d y^{2}}=-4 \pi \rho,
\end{aligned}
$$

where both $\rho$ and $j_{x}$ are functions of $A$ and $\phi$ only. Equation (2.12) was not included in Mart'yanov et al. (2008), which made their analysis inconsistent. For spatially periodic solutions $A(y+L)=A(y)$ and $\phi(y+L)=\phi(y)$, the global requirements of zero total charge and current are automatically satisfied. Indeed,

$$
4 \pi \int_{y}^{y+L} j_{x}\left(y^{\prime}\right) d y^{\prime}=-\int_{y}^{y+L} \frac{d^{2} A}{d y^{\prime 2}} d y^{\prime}=\frac{d A}{d y}{ }_{y}-\frac{d A}{d y}{ }_{y+L}=0
$$

and similarly for $\rho$. In the case of a solitary filament (current sheet) we should require $d \phi / d y \rightarrow 0, d A / d y \rightarrow 0$ for $y \rightarrow \pm \infty$, which also ensures validity of the same global restrictions.

For cold distributions the representation in terms of the distribution function is not convenient. Cold distributions (denoted by subscript ' $c$ ') describe particles which move along $x$ with the constant velocity $\beta_{c}$, so that $u_{x}=\beta_{c}\left(1-\beta_{c}^{2}\right)^{-1 / 2}, u_{y}=u_{z}=0$, which requires $d \phi / d y=\beta_{c}(d A / d y)$, or $\beta_{c}=(d \phi / d A)$, provided $|d \phi / d A|<1$. In this case $n_{c}=n(\phi, A)$ and

$$
\rho_{c}=q_{c} n_{c}, \quad j_{x, c}=q_{c} n_{c} \beta_{c} .
$$

As an example of a hot distribution let us consider a relativistic shifted Maxwellian (Juttner distribution), which will be extensively used throughout the paper:

$$
\begin{aligned}
f(H, P) & =C \exp [-m(h-\beta U) / T] \\
& =C \exp \left[-m\left(\gamma-\beta u_{x}\right) / T\right] \exp [-q(\phi-\beta A) / T] .
\end{aligned}
$$

It is worth mentioning that the invariant rest frame temperature is $T_{i n v}=\bar{\gamma} T$, where $\bar{\gamma}=\left(1-\beta^{2}\right)^{-1 / 2}$. In what follows, we shall consider a mixture of a cold distribution with a number of Juttner distributions (denoted by index ' $s$ '), so that

$$
\begin{aligned}
\rho & =\sum_{s} n_{s} q_{s} e^{-q_{s}\left(\phi-\beta_{s} A\right) / T_{s}}+q_{c} n_{c} \\
j_{x} & =\sum_{s} n_{s} q_{s} \beta_{s} e^{-q_{s}\left(\phi-\beta_{s} A\right) / T_{s}}+q_{c} n_{c} \beta_{c} \\
n_{s} & =C \int e^{-m_{s}\left(\gamma-\beta_{s} u_{x}\right) / T_{s}} d^{3} \boldsymbol{u} \\
n_{s} \beta_{s} & =C \int v_{x} e^{-m_{s}\left(\gamma-\beta_{s} u_{x}\right) / T_{s}} d^{3} \boldsymbol{u} .
\end{aligned}
$$


Here $\beta_{s}<1$. It is worth noting that the charge and current density for Maxwell distributed particles can be written as follows:

$$
\begin{aligned}
4 \pi \rho & =-\frac{\partial U}{\partial \phi}, \\
4 \pi j_{x} & =\frac{\partial U}{\partial A}, \\
U & =4 \pi \sum_{s} n_{s} T_{s} e^{-q_{s}\left(\phi-\beta_{s} A\right) / T_{s}},
\end{aligned}
$$

so that

$$
\begin{aligned}
\frac{d^{2}}{d y^{2}} \phi & =\frac{\partial U}{\partial \phi}, \\
\frac{d^{2}}{d y^{2}} A & =-\frac{\partial U}{\partial A} .
\end{aligned}
$$

The generally accepted approach to the analysis of nonlinear waves and structures is to derive an equation of the form

$$
\frac{d \boldsymbol{R}}{d X}=-\frac{\partial U}{\partial \boldsymbol{R}} .
$$

This equation can be interpreted as an equation of a particle motion in a potential. Here $X$ is the 'pseudotime', $\boldsymbol{R}$ is the 'pseudoposition vector' and $U$ is the 'pseudopotential'. Since (2.24) and (2.25) do not have the required structure, no 2D pseudopotential can be constructed.

\section{Electromagnetic filaments}

In this section we restrict ourselves to a special kind of solutions, $\phi=\bar{\beta} A, \bar{\beta}<1$. Physically, this means that in the frame moving with the velocity $\bar{\beta}$, there is no scalar potential. This condition is unavoidable if one of the distributions (and only one) describes particles, which are cold and, hence, move with the same speed along the current. Let these be ions moving with the velocity $\bar{\beta}$, then

$$
\frac{d p_{y}}{d t}=q\left(E_{y}-\bar{\beta} B_{z}\right)=0,
$$

which requires $E_{y}=\bar{\beta} B_{z}$ and, therefore, $\phi=\bar{\beta} A$.

Together with (2.11) and (2.12) this imposes the condition $\rho=\bar{\beta} j_{x}$, or

$$
\sum_{s} q_{s} \int\left(1-\bar{\beta} \beta_{x}\right) f_{s}\left(u_{x}+\left(q_{s} / m_{s}\right) A, \gamma+\left(q_{s} / m_{s}\right) \bar{\beta} A\right) d^{3} \boldsymbol{u}+q_{c} n_{c}\left(1-\bar{\beta}^{2}\right)=0,
$$

where we used $\beta_{c}=d \varphi / d A=\bar{\beta}$ and (2.14) is taken into account. Since in this case $j_{x}=j_{x}(A)$, one has a pseudo-potential type of equation

$$
\begin{aligned}
& \frac{d^{2} A}{d y^{2}}=-4 \pi j_{x} \equiv-\frac{d \Phi}{d A}, \\
& \frac{1}{2}\left(\frac{d A}{d y}\right)^{2}+\Phi(A)=\mathscr{E}=\text { const. }
\end{aligned}
$$


We are seeking for spatially periodic solutions. For this to occur, the pseudo-potential must have a stable minimum point. Without the loss of generality we shall calibrate the vector potential $A$ so that $d \Phi / d A=4 \pi j_{x}=0$ at $A=0$ :

$$
\sum_{s} \int q_{s} f_{s}\left(u_{x}, \gamma\right) d^{3} \boldsymbol{u}+q_{c} n_{c}(0)=0 .
$$

Here $n_{c}(0)$ is the value of the cold ion density (depending on $y$ implicitly via explicit dependence on $A$ ) in the point where the total charge density vanishes.

The requirement of stability is $d^{2} \Phi / d A^{2}>0$ at $A=0$, or $d \rho / d A>0$ at $A=0$. The latter can be further rewritten as follows:

$$
\sum_{s} \frac{q_{s}^{2}}{m_{s}} \int\left[\left(\partial f_{s} / \partial U_{s}\right)+\bar{\beta}\left(\partial f_{s} / \partial h_{s}\right)\right]_{A=0} d^{3} \boldsymbol{u}+q_{c}\left(\frac{d n_{c}}{d A}\right)_{A=0}>0 .
$$

For the cold particles, (3.2) gives

$$
q_{c} n_{c}=-\bar{\gamma}^{2} \sum_{s} q_{s} \int\left(1-\bar{\beta} \beta_{x}\right) f_{s}\left(u_{x}+\left(q_{s} / m_{s}\right) A, \gamma+\left(q_{s} / m_{s}\right) \bar{\beta} A\right) d^{3} \boldsymbol{u},
$$

and further, taking (2.10) into account

$$
\rho=\sum_{s} q_{s} \bar{\gamma}^{2} \bar{\beta} \int\left(\bar{\beta}-\beta_{x}\right) f_{s}\left(u_{x}+\left(q_{s} / m_{s}\right) A, \gamma+\left(q_{s} / m_{s}\right) \bar{\beta} A\right) d^{3} \boldsymbol{u},
$$

and the stability condition takes the form

$$
\sum_{s} \frac{q_{s}^{2}}{m_{s}} \int\left(\bar{\beta}-\beta_{x}\right)\left[\left(\partial f_{s} / \partial U_{s}\right)+\bar{\beta}\left(\partial f_{s} / \partial h_{s}\right)\right]_{A=0} d^{3} \boldsymbol{u}>0 .
$$

For the relativistic shifted Maxwellians $f_{s}=C_{s} \exp \left[-m_{s}\left(\gamma-\beta_{s} u_{x}\right) / T_{s}\right) \exp \left[-q_{s}(\bar{\beta}-\right.$ $\left.\left.\beta_{s}\right) A / T_{s}\right]$, the relation (3.9) can be written as

$$
\sum_{s} \frac{q_{s}^{2}\left(\beta_{s}-\bar{\beta}\right)^{2}}{T_{s}} n_{s}>0
$$

which is always satisfied, while (3.7) takes the form

$$
q_{c} n_{c}=-\overline{\gamma^{2}} \sum_{s}\left(1-\bar{\beta} \beta_{s}\right) q_{s} n_{s} e^{-q_{s}\left(\bar{\beta}-\beta_{s}\right) A / T_{s}} .
$$

\subsection{Solutions without backstreaming particles}

In order to elucidate the role of the backstreaming particles we analyze below two simple cases of the species moving in the same direction. Let us consider first the case of cold ions and Maxwellian electrons. Let the cold ions move with the velocity $\beta_{i}$. This requires $\phi=\beta_{i} A$. Choosing the electron Maxwellian shifted around $\beta_{e} \neq \beta_{i}$, one has $\left(q_{i}=-q_{e}=q\right)$

$$
\begin{aligned}
\frac{d^{2}}{d y^{2}} A & =-4 \pi q\left(n_{i} \beta_{i}-n_{e} \beta_{e} e^{q\left(\beta_{i}-\beta_{e}\right) A / T}\right), \\
\frac{d^{2}}{d y^{2}} \phi & =-4 \pi q\left(n_{i}-n_{e} e^{q\left(\beta_{i}-\beta_{e}\right) A / T}\right) .
\end{aligned}
$$


The requirement $\phi=\beta_{i} A$ gives

$$
\begin{aligned}
& n_{i}-n_{e} e^{-q_{e}\left(\beta_{i}-\beta_{e}\right) A / T}=\beta_{i}\left(n_{i} \beta_{i}-n_{e} \beta_{e} e^{q\left(\beta_{i}-\beta_{e}\right) A / T}\right), \\
n_{i} & =n_{e} \gamma_{i}^{2}\left(1-\beta_{i} u_{e}\right) e^{q\left(\beta_{i}-\beta_{e}\right) A / T}, \\
\frac{d^{2}}{d y^{2}} A & =-4 \pi q n_{e}\left[\beta_{i} \gamma_{i}^{2}\left(1-\beta_{i} \beta_{e}\right)-\beta_{e}\right] e^{q\left(\beta_{i}-\beta_{e}\right) A / T} \\
& =-4 \pi q n_{e}\left(\beta_{i}-\beta_{e}\right) \gamma_{i}^{2} e^{q\left(\beta_{i}-\beta_{e}\right) A / T} .
\end{aligned}
$$

We have arrived at the well-known equation for Harris equilibrium. The solution of this equation describes a single current layer.

Let now both species (ions and electrons) be Maxwellian, then

$$
\begin{aligned}
& \rho=q\left(n_{i} e^{-q\left(\beta-\beta_{i}\right) A / T_{i}}-n_{e} e^{q\left(\beta-\beta_{e}\right) A / T_{e}}\right), \\
& j_{x}=q\left(n_{i} \beta_{i} e^{-q\left(\beta-\beta_{i}\right) A / T_{i}}-n_{e} \beta_{e} e^{q\left(\beta-\beta_{e}\right) A / T_{e}}\right), \\
& j_{x}=\beta \rho \Rightarrow \\
& n_{i}\left(1-\beta \beta_{i}\right) e^{-q\left(\beta-\beta_{i}\right) A / T_{i}}=n_{e}\left(1-\beta \beta_{e}\right) e^{q\left(\beta-\beta_{e}\right) A / T_{e}},
\end{aligned}
$$

and therefore $-\left(\beta-\beta_{i}\right) / T_{i}=\left(\beta-\beta_{e}\right) / T_{e}$ so that

$$
\beta=\left(T_{e} \beta_{i}+T_{i} \beta_{e}\right) /\left(T_{i}+T_{e}\right),
$$

and we again arrive at the Harris equilibrium. The solution of this equation also describes a single current layer. In neither case we arrive at multi-layered structure. This means that backstreaming particles are necessary for a filamentary structure.

\subsection{Cold ions with three hot species}

Let us now consider a self-consistent solution with four species: (a) cold forward streaming ions $\beta_{i f}=\beta>0$, (b) Maxwellian forward streaming electrons $\beta_{\text {ef }}>0$, (c) Maxwellian backstreaming ions $\beta_{i b}<0$ and (d) Maxwellian backstreaming electrons $\beta_{e b}<0$. Apparently, this is a minimal required set to allow for spatially periodic patterns (see above). Now for each Maxwellian species $s=e f, e b, i b$

$$
n_{(s)}=n_{s} e^{c_{s} A}, \quad c_{s}=q_{s}\left(\beta-\beta_{s}\right) / T_{s},
$$

while $n_{i f}=n_{i f}(A)$ for cold forward streaming ions. Here $q_{i f}=q_{i b}=q, q_{e f}=q_{e b}=-q$. The charge and current density are, respectively,

$$
\begin{aligned}
& \rho=q n_{i f}+\sum_{s} q_{s} n_{s} e^{-c_{s} A}, \\
& j=q n_{i f} \beta+\sum_{s} q_{s} n_{s} \beta_{s} e^{-c_{s} A} .
\end{aligned}
$$

The requirement $\phi=\beta A$ gives $\rho=\beta j$ and, therefore,

$$
n_{i f}=-\gamma^{2} \sum_{s}\left(1-\beta \beta_{s}\right)\left(q_{s} / q\right) n_{s} e^{-c_{s} A} .
$$


The total charge density, respectively, is

$$
\rho=-\sum_{s} q_{s} \beta\left(\beta-\beta_{s}\right) \gamma^{2} n_{s} e^{-c_{s} A} .
$$

We calibrate the potentials so that $A=0 \Rightarrow \rho=0$, which gives

$$
\begin{aligned}
& \sum_{s} q_{s}\left(\beta-\beta_{s}\right) n_{s}=0 \Rightarrow \\
& n_{i b}=\frac{n_{e f}\left(\beta-\beta_{e f}\right)+n_{e b}\left(\beta-\beta_{e b}\right)}{\beta-\beta_{i b}} .
\end{aligned}
$$

In other words, the vector potential and the scalar potential are both zero in the point $y$, where the charge density is zero.

The equation for $A$ now reads

$$
\frac{d^{2} A}{d x^{2}}=4 \pi \gamma^{2} \sum_{s} q_{s}\left(\beta-\beta_{s}\right) n_{s} e^{-c_{s} A},
$$

where $s=e f, e b, i b$. This equation can be further rewritten in the form

$$
\begin{aligned}
\frac{d^{2} A}{d x^{2}} & =-\frac{d U}{d A} \\
U & =4 \pi \gamma^{2} \sum_{s} \frac{q_{s}\left(\beta-\beta_{s}\right) n_{s}}{c_{s}} e^{-c_{s} A} \\
& =4 \pi \gamma^{2} \sum_{s} n_{s} T_{s} e^{-c_{s} A}
\end{aligned}
$$

Since $U(A \rightarrow \pm \infty) \rightarrow \infty$, any solution of this equation describes a spatially periodic system of current sheets (filaments), provided $n_{i f}>0$.

It is of interest to study small deviations $c_{S} A \ll 1$, for which

$$
\begin{aligned}
U & =U_{0}+\frac{1}{2} K^{2} A^{2}, \\
U_{0} & =4 \pi \gamma^{2} \sum_{s} n_{s} T_{s}, \\
K^{2} & =\sum_{s} \frac{4 \pi \gamma^{2} n_{s} q_{s}^{2}\left(\beta-\beta_{s}\right)\left(\beta-\beta_{s}\right)}{T_{s}} .
\end{aligned}
$$


It is easy to see that $1 / K$ plays the role of the generalized Debye length for the relativistic streaming equilibria under consideration. Respectively,

$$
\begin{aligned}
A= & A_{m} \cos (K y), \\
n_{(e f)}= & n_{e f}\left[1+q\left(\beta-\beta_{e f}\right) A / T_{e f}\right], \\
n_{(e b)}= & n_{e b}\left[1+q\left(\beta-\beta_{e b}\right) A / T_{e b}\right], \\
n_{(i b)}= & n_{i b}\left[1-q\left(\beta-\beta_{i b}\right) A / T_{i b}\right], \\
n_{(i f)}= & -\gamma^{2} \sum_{s}\left(1-\beta \beta_{s}\right)\left(q_{s} / q\right) n_{s} \\
& +\left[\gamma^{2} q \sum_{s}\left(1-\beta \beta_{s}\right) n_{s}\left(\beta-\beta_{s}\right) / T_{s}\right] A .
\end{aligned}
$$

If

$$
\begin{aligned}
n_{i f} \equiv n_{i}(A=0) & =-\gamma^{2} \sum_{s}\left(1-\beta \beta_{s}\right)\left(q_{s} / q\right) n_{s} \\
& =\frac{n_{e f}\left(\beta_{e f}-\beta_{i b}\right)+n_{e b}\left(\beta_{e b}-\beta_{i b}\right)}{\beta-\beta_{i b}}>0,
\end{aligned}
$$

then there always exists $A_{m}$ such that for $|A|<A_{m}$ the ion density $n_{i}>0$. This inequality is always satisfied for $\left|\beta_{i b}\right|>\left|\beta_{e b}\right|$, while for $\left|\beta_{i b}\right|<\left|\beta_{e b}\right|$ there is the restriction

$$
n_{e b}<n_{e f}\left(\beta_{e f}+\left|\beta_{i b}\right|\right) /\left(\left|\beta_{e b}\right|-\left|\beta_{i b}\right|\right) .
$$

The condition $c_{s} A \lesssim 1$ requires that $\left(\beta+\left|\beta_{b}\right|\right) q A_{m} / T_{b} \lesssim 1$ and $\left(\beta-\left|\beta_{e f}\right|\right) q A_{m} /$ $T_{e f} \lesssim 1$, which means

$$
\begin{aligned}
& q A_{m} / T_{i b} \sim q A_{m} / T_{e b} \lesssim 0.5, \\
& q A_{m} / T_{e f} \lesssim 1 /\left(\beta-\beta_{e f}\right) .
\end{aligned}
$$

The last relation allows $q A_{m} / T_{e f} \gg 1$ for $\beta-\beta_{e f} \ll 1$. We also see that the temperature of the backstreaming particles is of the same order as the potential in the filament.

For the visualization below, we have chosen the following normalized parameters: $\beta=0.92, \beta_{e f}=0.91, \beta_{i b}=-0.9, \beta_{e b}=-0.8, A_{m}=0.2, A=A_{m} \cos (y), T_{i b}=2, T_{e b}=1$, $T_{e f}=0.1, n_{i b}=n_{e b}=1$. Figure 1 illustrates the transverse cut of the $2 \mathrm{D}$ filamentary structure. It is seen that the fluctuations of the density of the forward streaming cold ions are much stronger than the fluctuations of the densities of hot species. The filaments are charged positively. The densities of the cold ions are higher in the region of positive potential, while the densities of hot other species are higher in the potential well.

\subsection{Four hot species}

Let us now consider four Maxwellian species in an electromagnetic filament. It will be more transparent here to perform the analysis in the frame where there is no 

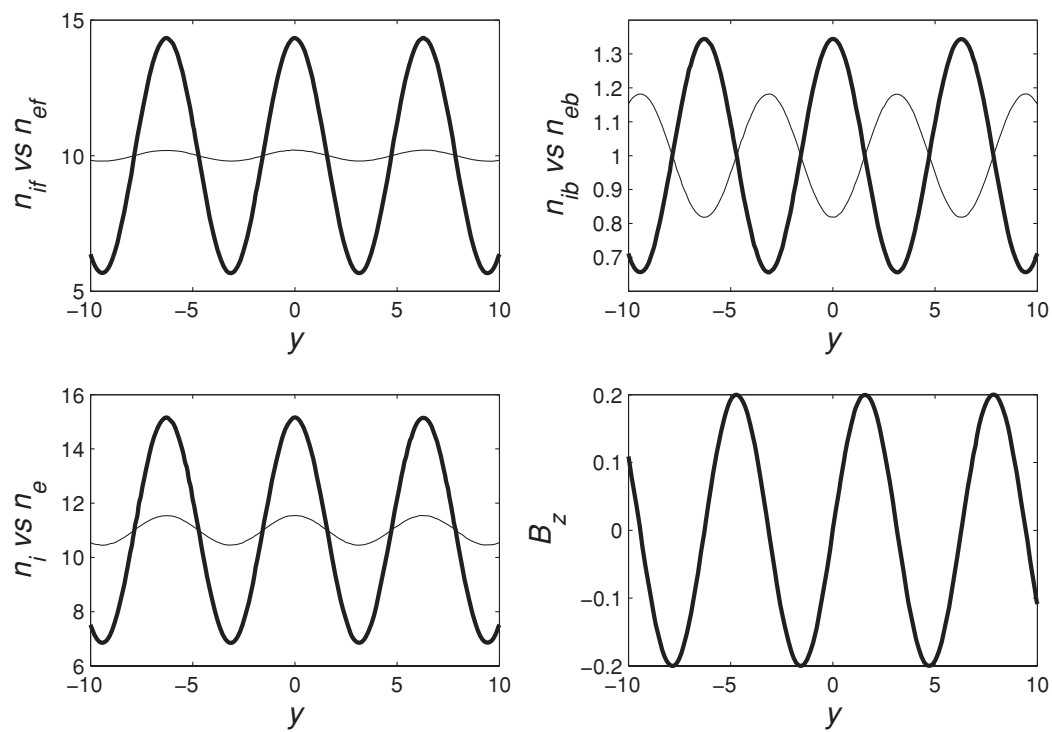

Figure 1. Structure of a filament. Left top: Densities of the forward streaming particles across the filament. Right top: Densities of the backstreaming particles. Left bottom: Total densities. Thick lines are for electrons. Right bottom: Magnetic field.

potential, that is $\beta=0$. This requires

$$
\begin{gathered}
\sum_{s} n_{s} q_{s} e^{q_{s} \beta_{s} A / T_{s}}=0, \\
n_{i f} e^{q \beta_{i f} A / T_{i f}}+n_{i b} e^{-q\left|\beta_{i b}\right| A / T_{i b}}-n_{e f} e^{-q \beta_{e f} A / T_{e f}}-n_{e b} e^{q\left|\beta_{e b}\right| A / T_{e b}}=0 .
\end{gathered}
$$

The latter expression can be made identically zero if

$$
\begin{array}{ll}
T_{e b}=\frac{\left|\beta_{e b}\right| T_{i f}}{\beta_{i f}}, & n_{i f}=n_{e b} . \\
T_{e f}=\frac{\beta_{e f} T_{i b}}{\left|\beta_{i b}\right|}, & n_{e f}=n_{i b} .
\end{array}
$$

With this condition taken into account, the current density is

$$
\begin{aligned}
j_{x} & =\sum_{s} n_{s} q_{s} e^{q_{s} \beta_{s} A / T_{s}} \\
& =q\left[n_{i f}\left(\beta_{i f}+\left|\beta_{e b}\right|\right) e^{q \beta_{i f} A / T_{i f}}-n_{i b}\left(\beta_{e f}+\left|\beta_{i b}\right|\right) e^{-q\left|\beta_{i b}\right| A / T_{i b}}\right]
\end{aligned}
$$

so that the pseudopotential takes the form

$$
\Phi=4 \pi\left[\frac{n_{i f}\left(\beta_{i f}+\left|\beta_{e b}\right|\right) T_{i f}}{\beta_{i f}} e^{q \beta_{i f} A / T_{i f}}+\frac{n_{i b}\left(\beta_{e f}+\left|\beta_{i b}\right|\right) T_{i b}}{\left|\beta_{i b}\right|} e^{-q\left|\beta_{i b}\right| A / T_{i b}}\right] .
$$

Since $\Phi \rightarrow \infty$ for $A \rightarrow \pm \infty$ and has only one minimum, the equation for $A$ has only spatially periodic solutions. There are no additional constraints on the parameters of different species. 


\section{Potential filaments}

If no distribution is cold, the relation $\phi=\beta A$ is no longer necessary (since $E_{y} / B_{z}=$ construction does not have to be satisfied), which means that there may not be a frame where the electric potential vanishes. It is difficult to treat the problem in the fully nonlinear regime, but it is possible to advance in the case of weak filaments, $q A / T \ll 1, q \phi / T \ll 1$. In a more general way, we shall assume that it is possible to expand (for each species, index $s$ omitted for brevity)

$$
\begin{aligned}
f\left(U, h, u_{z}\right)= & f\left(u_{x}, \gamma, u_{z}\right)+(q A / m) \frac{\partial f}{\partial u_{x}}+(q \phi / m)\left(\frac{\partial f}{\partial \gamma}\right) \\
& +\frac{1}{2}(q A / m)^{2}\left(\frac{\partial^{2} f}{\partial u_{x}^{2}}\right)+\frac{1}{2}(q \phi / m)^{2}\left(\frac{\partial^{2} f}{\partial \gamma^{2}}\right) \\
& +(q \phi / m)(q A / m)^{2}\left(\frac{\partial^{2} f}{\partial u_{x} \partial \gamma}\right)+\ldots
\end{aligned}
$$

Then

$$
\begin{aligned}
\rho= & \sum n_{s} q_{s} \int f\left(u_{x}, \gamma, u_{z}\right) d^{3} \boldsymbol{u}+A \sum_{s} \frac{n_{s} q_{s}^{2}}{m_{s}} \int \frac{\partial f_{s}}{\partial u_{x}} d^{3} \boldsymbol{u} \\
& +\phi \sum_{s} \frac{n_{s} q_{s}^{2}}{m_{s}} \int \frac{\partial f_{s}}{\partial \gamma} d^{3} \boldsymbol{u}, \\
j_{x}= & \sum n_{s} q_{s} \int \frac{u_{x}}{\gamma} f\left(u_{x}, \gamma, u_{z}\right) d^{3} \boldsymbol{u}+\sum A \sum_{s} \frac{n_{s} q_{s}^{2}}{m_{s}} \int \frac{u_{x}}{\gamma} \frac{\partial f_{s}}{\partial u_{x}} d^{3} \boldsymbol{u} \\
& +\phi \sum_{s} \frac{n_{s} q_{s}^{2}}{m_{s}} \int \frac{u_{x}}{\gamma} \frac{\partial f_{s}}{\partial \gamma} d^{3} \boldsymbol{u},
\end{aligned}
$$

where we restricted ourselves with the lowest order. Although the expansion can be carried out for arbitrary distributions, it is more physically clear with the distributions described by the single-temperature parameter, like the relativistic Maxwellian (Juttner). We will limit our analysis with this case, where

$$
\begin{aligned}
& \rho=\sum_{s} n_{s} q_{s}\left[1-\frac{q_{s}\left(\phi-\beta_{s} A\right)}{T_{s}}+\frac{1}{2}\left(\frac{q_{s}\left(\phi-\beta_{s} A\right)}{T_{s}}\right)^{2}\right], \\
& j_{x}=\sum_{s} n_{s} q_{s} \beta_{s}\left[1-\frac{q_{s}\left(\phi-\beta_{s} A\right)}{T_{s}}+\frac{1}{2}\left(\frac{q_{s}\left(\phi-\beta_{s} A\right)}{T_{s}}\right)^{2}\right] .
\end{aligned}
$$

In the lowest order, linear approximation, one has

$$
\begin{gathered}
\rho=A \sum_{s} \frac{n_{s} q_{s}^{2} \beta_{s}}{T_{s}}-\phi \sum_{s} \frac{n_{s} q_{s}^{2}}{T_{s}}, \\
j_{x}=A \sum_{s} \frac{n_{s} q_{s}^{2} \beta_{s}^{2}}{T_{s}}-\phi \sum_{s} \frac{n_{s} q_{s}^{2} \beta_{s}}{T_{s}},
\end{gathered}
$$


where we also applied the conditions $\sum_{s} n_{s} q_{s}=0, \sum_{s} n_{s} q_{s} \beta_{s}=0$. Substituting this into (2.11) and (2.12) one gets

$$
\begin{aligned}
\frac{d^{2} A}{d y^{2}} & =-K_{2} A+K_{1} \phi, \\
\frac{d^{2} \phi}{d y^{2}} & =-K_{1} A+K_{0} \phi, \\
K_{0} & =\sum_{s} \frac{4 \pi n_{s} q_{s}^{2}}{T_{s}}>0, \\
K_{1} & =\sum_{s} \frac{4 \pi n_{s} q_{s}^{2} \beta_{s}}{T_{s}}, \\
K_{2} & =\sum_{s} \frac{4 \pi n_{s} q_{s}^{2} \beta_{s}^{2}}{T_{s}}>0, \quad K_{2}<K_{0},
\end{aligned}
$$

which has the solutions $A, \phi \propto e^{i k y}$, where

$$
\begin{gathered}
\left(K_{0}+k^{2}\right)\left(K_{2}-k^{2}\right)=K_{1}^{2} \\
k^{2}=\frac{1}{2}\left[K_{2}-K_{0} \pm \sqrt{\left(K_{0}+K_{2}\right)^{2}-4 K_{1}^{2}}\right] .
\end{gathered}
$$

Since

$$
K_{0} K_{2}-K_{1}^{2}=\sum_{i j} \frac{n_{i} n_{j} q_{i}^{2} q_{j}^{2}\left(\beta_{i}-\beta_{j}\right)^{2}}{2 T_{i} T_{j}}>0
$$

the solution

$$
k^{2}=\frac{1}{2}\left[K_{2}-K_{0}+\sqrt{\left(K_{0}+K_{2}\right)^{2}-4 K_{1}^{2}}\right]>0
$$

corresponds to the periodic filaments.

\section{Conclusions and discussion}

Two-dimensional filamentary equilibria are, in fact, periodically arranged current sheets and, therefore, could be expected to be described in a way similar to what has been done for a relativistic periodic Harris sheet structure. Indeed, the distribution functions are immediately expressed in terms of the integrals of motion, which allow to express the charge and current density as functions of the scalar and vector potential only. It is worth mentioning that, in general, the scalar potential, and, therefore, the electric field, cannot be ignored unless special conditions are imposed. In order to satisfy these conditions, the species' densities, temperatures and velocities should be fine tuned, thus reducing the number of free parameters. This means that for arbitrarily chosen initial conditions, the developed filamentary structure will not be necessarily non-potential or even stationary.

To summarize: 
- A non-potential filamentary equilibrium with one cold ion distributions and three hot species is constructed and necessary conditions for such equilibrium are established.

- A non-potential filamentary equilibrium with four relativistic Maxwellian species is constructed and necessary conditions for such equilibrium are established.

- The role of the backstreaming distributions in the formation of stationary spatially periodic filamentary structure is elucidated and it is shown that at least four streaming species are necessary for the existence of non-potential filaments.

- A weak filamentary equilibrium with potential is derived for arbitrary number of Maxwellian species.

Analysis of fully nonlinear filaments with electrostatic potential appears to be difficult and will be discussed elsewhere.

\section{Acknowledgements}

The work is partially supported by the ISSI grant 'Relativistic reconnection and collisionless shocks'. M. G. and A. S. are partly supported by BSF grant no. 2006095. M. M. is partly supported by NSF grant AST-0708213, NASA ATFP grant NNX08AL39G and DOE grant DE-FG02-07ER54940.

\section{References}

Balikhin, M. and Gedalin, M. 2008 Generalization of the harris current sheet model for non-relativistic, relativistic and pair plasmas. J. Plasma Phys. 74, 749.

Bret, A. 2009 Weibel, two-stream, filamentation, oblique, bell, buneman...which one grows faster? Astrophys. J. 699, 990.

Frederiksen, J., Hededal, C. and Haugbolle, T. 2004 Magnetic field generation in collisionless shocks: pattern growth and transport. Astrophys. J. Lett. 608, L13.

Hededal, C. and Nishikawa, K. 2005 The influence of an ambient magnetic field on relativistic collisionless plasma shocks. Astrophys. J. Lett. 623, L89.

Lyubarsky, Y. and Eichler, D. 2006 Are gamma-ray burst shocks mediated by the weibel instability? Astrophys. J. 647, 1250.

Mart'yanov, V. Y., Kocharovsky, V. V. and Kocharovsky, V. V. 2008 Saturation of relativistic weibel instability and the formation of stationary current sheets in collisionless plasma. JETP 107, 1049.

Medvedev, M. 2007 Weibel turbulence in laboratory experiments and grb/sn shocks. Astrophys. Space Sci 307, 245.

Medvedev, M. and Loeb, A. 1999 Generation of magnetic fields in the relativistic shock of gamma-ray burst sources. Astrophys. J. 526, 697.

Nishikawa, K.-I., Niemiec, J., Hardee, P. E., Medvedev, M., Sol, H., Mizuno, Y., Zhang, B., Pohl, M., Oka, M. and Hartmann, D. H. 2009 Weibel instability and associated strong fields in a fully three-dimensional simulation of a relativistic shock. Astrophys. J. Lett. 698, L10.

Pegoraro, F., Bulanov, S., Califano, F. and Lontano, M. 1996 Nonlinear development of the weibel instability and magnetic field generation in collisionless plasmas. Physica Scripta T63, 262.

Schaefer-Rolffs, U. and Tautz, R. C. 2008 The relativistic kinetic weibel instability: comparison of different distribution functions. Phys. Plasmas 15, 2105. 
Silva, L. O., Fonseca, R. A., Tonge, J. W., Dawson, J. M., Mori, W. B. and Medvedev, M. V. 2003 Interpenetrating plasma shells: near-equipartition magnetic field generation and nonthermal acceleration. Astrophys. J. 596, L121.

Spitkovsky, A. 2008 On the structure of relativistic collisionless shocks in electron-ion plasmas. Astrophys. J. 673, L39.

Yoon, P. H. 2007 Relativistic weibel instability. Phys. Plasmas 14, 024504. 